are growing, information systems in most of these countries are not adequate to the task.

Objectives Building and Strengthening the Monitoring and Evaluation System for Maternal and Newborn interventions in developing countries experience sharing

Methods Makerere University School of Public Health implemented a Maternal and Newborn study in Eastern Uganda. This was based on Quasi experimental design for program implementation. Data was collected from both intervention and control areas. Data was collected from newly delivered women, Community Health Workers (CHWs), and Health Facilities using both qualitative and quantitative data collection methods. We used both traditional and contemporary ME methodologies which include; Theory of Change, Logic Model among others.

Result The system helped in setting targets and strategies based on the community needs and tracking project implementation challenges. For example through CHWs reporting, we were able to identify newborn death which were not captured at the health facility. The $2014 \mathrm{CHWs}$ reports indicated total of 145 newborn deaths which were not reported in the district HMIS data. Additionally, CHWs reported 145 newborns with low birth weight. Though the short term surveys indicated an improved skilled delivery from $66 \%$ to $83 \%$, a considerate proportion (16.6\%) still deliver from homes or TBAs. Only $36.8 \%$ and $50 \%$ of women with small babies received information on how to care for small babies in the intervention and control area respectively. Additionally, only 5.3\% and 30\% received KMC in the intervention and control area respectively. Using Theory of Change methodology, we were able to understand health system challenges. For example, we found that although women were being referred to deliver from health facilities, the facilities lacked qualified health workers and they were using nursing assistants in conducting deliveries. Other challenges included having inadequate drugs and equipments for maternal health services. The ME moments gave us an opportunity for the service providers and the decision makers to interact and take appropriate decisions to address the above challenges. This also provided timely information to the key stakeholders on the provision of maternal and newborn care services in their respective communities. As a result, this helped in identifying the resources and available partners who can come in and help. For example, in one of the facilities, there was no a placenta pit but as a result of meeting the decision makers understood the need for placenta pit and the directives were made to avail money for construction.

Conclusion Having a comprehensive ME system for Maternal and Newborn interventions is very important because it helps in providing information to the key stakeholders at both levels thus holding them accountable and taking appropriate actions. Project should aim at putting in place ME systems that allow fully participation of stakeholders including the beneficiaries as well as providing timely information for evidence based decision making.

\section{STRENGTHENING MONITORING AND EVALUATION SYSTEM FOR MATERNAL AND NEWBORN INTERVENTIONS: A CASE STUDY OF MATERNAL AND NEWBORN STUDY IN EASTERN UGANDA}

Rornald Muhumuza Kananura. Makerere University School of Public Health, Kampala, Uganda

\subsection{6/bmjopen-2015-forum2015abstracts. 17}

Background Despite the billions of dollars spent in achieving millennium development goal 4 and 5 in developing countries, it seems not possible for most of the African countries to meet the target by 2015 . While demands for reliable and timely data 